\title{
APUNTES EN TORNO AL TLC CENTROAMÉRICA/ESTADOS UNIDOS Y SUS IMPLICACIONES PARA LA EDUCACIÓN SUPERIOR
}

\begin{abstract}
Resumen: El presente artículo tiene como propósito realizar una breve aproximación analítica a los aspectos que, en el marco de la propuesta de Tratado de Libre Comercio entre Centroamérica y Estados Unidos, conciernen o implican a la educación superior y, en especial, a la educación superior pública.

El artículo aborda dos ejes de análisis. El primero de ellos, está referido específicamente a los lineamientos y estipulaciones que involucra el texto del TLC Centroamérica/Estados Unidos, en lo relativo a las implicaciones que derivan de esa propuesta para las instituciones de educación superior y, en particular, para la educación superior pública. El segundo eje, por su parte, consiste en una breve revisión de trabajos elaborados por especialistas en torno a los alcances que, en su caso, presentan para la educación superior algunas de las estipulaciones contenidas en el Acuerdo General sobre Comercio de Servicios (AGCS), de la Organización Mundial del Comercio.
\end{abstract}

Palabras clave: LIBRE COMERCIO/ EDUCACIÓN SUPERIOR/ COMERCIO DE SERVICIOS/

ABSTRACT: This article intends to make a brief analysis about the repercussions of the Free Trade Agreement between Central America and The United States (CAFTA) on the aspects concerning to the Higher education; especially, public higher education.

There are two analysis approaches in this article. The first one deals specifically with the tendencies and regulations of the CAFTA in reference to its proposal to the high educational field. The second approach refers to a brief literary review about some specialists' works related to the consequences to the higher education of some regulations contained in the Service Trade General Agreement by the World Commerce Organization.

Key Words: FREE TRADE/ HIGHER EDUCATION/ SERVICE COMMERCE/

\section{Presentación}

En la óptica de la Comisión Económica para América Latina y el Caribe (CEPAL), la globalización comporta una "naturaleza dramática". Esta deriva de los acelerados cambios que en el tiempo y en el espacio acontecen como resultado de los dinámicos movimientos y desplazamientos posibilitados por los espacios instrumentales abiertos por las realizaciones de las tecnologías de la información y la comunicación: "los avances tecnológicos, sobre todo en las comunicaciones y en la microelectrónica, han cambiado radicalmente la manera en que las naciones interactúan entre sí." (Rosenthal, 1998, p. 2)

\footnotetext{
${ }^{1}$ Licenciatura en Filosofía de la Universidad de Costa Rica; Maestría en Ciencias con Especialidad en Investigación Educativa del Departamento de Investigaciones Educativas, Centro de Investigaciones y Estudios Avanzados del Instituto Politécnico Nacional, México; Asesor de Rectoría, Universidad de Costa Rica.
}

Correo electrónico: Imunoz@rectoria.ucr.ac.cr

Artículo recibido: 21 de abril, 2005

Aprobado: 28 de abril, 2005 
En sus significaciones medulares, estos procesos y su impronta de cambio representan la emergencia de nuevas perspectivas y escenarios para el desarrollo de los procesos económico/sociales y "representan transformaciones cualitativas con respecto al pasado." (CEPAL, 2000, p. 13). En su significado para los contextos del mercado, el intercambio y la movilidad de los bienes económicos, destaca en este sentido la predominante importancia que adquieren la especialización de la producción, tanto como su descentralización geográfica en una perspectiva de proyección y escala mundiales.

A propósito de la educación superior, estos acontecimientos anclan su repercusión privilegiadamente en el escenario del proyecto de formación, en relación con los nuevos perfiles profesionales y de la fuerza de trabajo, que ahora establecen las demandas de sistemas económico-productivos cada vez más centrados en el uso y aplicación del conocimiento y de los insumos e instrumentos que proporcionan las realizaciones científico/tecnológicas.

Pero por otra parte, las condiciones para el desarrollo de la educación superior quedan inscritas en un escenario complejo cuyo marco de referencia trasciende las coordenadas de lo local y lo nacional. Además de las tendencias estructurales internas que se manifiestan en la escala de lo nacional, tales, por ejemplo, como la de la eclosión de la demanda de los servicios de educación superior, hacen su aparición igualmente otros fenómenos durante los últimos años, especialmente aquellos que están "asociados a la emergencia de una vigorosa globalización del conocimiento, un mercado educativo sin fronteras, nuevos proveedores $y$, con ellos, nuevos modelos de oferta educativa." (García Guadilla, 2003, p. 17)

Las implicaciones que el texto del Tratado de Libre Comercio entre Centroamérica y Estados Unidos presenta para la educación superior pública en los países del istmo, constituye un tema apenas aún por asumir y explorar. De igual manera, está pendiente también de desarrollar entre las comunidades académicas y entre las autoridades institucionales de la educación superior en los países de Centroamérica, la discusión acerca de las implicaciones que en este mismo sentido se desprenden de los avances de negociación cristalizados en los acuerdos suscritos por los países miembros de la Organización Mundial del Comercio (OMC), en el marco del Acuerdo General sobre Comercio de Servicios (AGCS).

El presente artículo constituye lo que, de momento, puede ser considerado como un ejercicio de aproximación apenas muy breve y muy introductorio, a manera de apuntes para la discusión. Su propósito consiste en identificar algunas de las principales zonas y configuraciones de inquietud y duda que, en cada caso, ambos escenarios y procesos 
mencionados generan, a partir o desde un referente de lectura anclado en las coordenadas de una perspectiva de educación superior pública. Así, quedan pendientes de abordar en su especificidad, aspectos sustantivos tales como el de las implicaciones que el TLC presenta en materia de sistemas de evaluación y acreditación de la educación superior. Estos aspectos constituyen parte de los pendientes cuyo análisis habrá que asumir en una próxima oportunidad.

\section{TLC Centroamérica/Estados Unidos: comercio de servicios y educación superior}

En el texto de la propuesta de TLC entre Centroamérica y Estados Unidos, el capítulo once, intitulado "Comercio Transfronterizo de Servicios", señala las coordenadas de Fundamentación contractual en las que tendría que llevarse a cabo el comercio de servicios, y se refiere "a las medidas que adopte o mantenga una Parte que afecten al comercio transfronterizo de servicios por un proveedor de servicios de otra Parte. (SICE, 2004, p. 111). Estas medidas involucran las medidas que afecten a:

(a) la producción, distribución, comercialización, venta y suministro de un servicio;

(b) la compra o uso de, o el pago por, un servicio;

(c) el acceso a y el uso de sistemas de distribución y transporte, o de redes de telecomunicaciones y los servicios relacionados con el suministro de un servicio;

(d) la presencia en su territorio de un proveedor de servicios de otra Parte; y

(e) el otorgamiento de una fianza u otra forma de garantía financiera, como condición para la prestación de un servicio." (SICE, 2004, p. 11-1)

No sería el espacio del presente breve y puntual artículo, el lugar apropiado para hacer un análisis específico con respecto a los alcances de los elementos que involucra el anterior cuadriculado de disposiciones. El propósito aquí es únicamente indicarlos, bajo el interés por establecer un marco de referencia que nos permita ubicar las implicaciones que el TLC pueda tener y que, como se verá, de hecho plantea en diverso sentido para la educación superior.

En el inciso sexto del artículo 11.1 de ese mismo capítulo once, se establece una especificación que delimita la aplicación del TLC en lo que concierne a una tipificación de los servicios susceptibles de ser comercializados. Textualmente, en dicho inciso se estipula 
que: "Este Capítulo no se aplica a los servicios suministrados en el ejercicio de facultades gubernamentales." Y, a continuación, se especifica en qué consiste un "servicio suministrado en el ejercicio de facultades gubernamentales": "Un "servicio suministrado en el ejercicio de facultades gubernamentales" significa todo servicio que no se suministre en condiciones comerciales ni en competencia con uno o varios proveedores de servicios." (SICE, 2004, p. 11-2)

Una apropiación inmediata del significado de lo anterior podría entrañar la idea de que todo tipo de servicio que se encuentre institucionalmente bajo la tutela o la administración del Estado, especialmente en lo que a la disposición financiera y a las características de su prestación se refiere, no formaría parte de los servicios considerados como de interés según la formulación normativa que se propone para regular las relaciones comerciales en el marco del TLC.

De este modo y como punto de partida, cabría asumir la presunción de que los servicios educativos suministrados por el Estado o a través de instituciones públicas, no quedan contemplados como parte de los servicios que en el TLC se incluyen y tipifican como susceptibles de comercialización. Esta apreciación, sin embargo, tal como se verá más adelante, es inexacta.

Desde el vértice de las delimitaciones procedimentales que traza el escenario de libre comercio, es igualmente importante tener en cuenta dos reglas fundamentales estipuladas como operadores de base que rigen la organización de las relaciones contractuales comerciales entre las partes. La primera de ellas es la regla de "Trato Nacional":

1. Cada Parte otorgará a los proveedores de servicios de otra Parte un trato no menos favorable que el que otorgue, en circunstancias similares, a sus propios proveedores de servicios.

2. El trato otorgado por una Parte de conformidad con el párrafo 1 significa, respecto a un gobierno de nivel regional, un trato no menos favorable que el trato más favorable que ese gobierno de nivel regional otorgue, en circunstancias similares, a los proveedores de servicios de la Parte de la que forma parte integrante. (SICE, 2004, p. 11-2)

Esta regla establece que bajo consideración de ningún tipo, un país puede otorgar condiciones preferenciales a proveedor alguno, tanto en el caso de que este proveedor sea un nacional o, como en el caso de Centroamérica, que esté situado en el espacio de cobertura de un marco de integración regional como el que, por ejemplo, configura el 
escenario del Sistema de la Integración Centroamericana (SICA). Las condiciones para la movilidad y puesta en oferta de las mercancías así como para la inversión comercial, deben ser, en todo caso, exactamente las mismas y por igual para todos los países signatarios del Tratado.

Una segunda regla importante es la así denominada regla de "Trato de Nación más Favorecida”. Esta regla involucra una disposición que sirve de complemento a la anterior: “

Cada Parte otorgará a los proveedores de servicios de otra Parte un trato no menos favorable que el que otorgue, en circunstancias similares, a los proveedores de servicios de cualquier otra Parte o de un país que no sea parte. (SICE, 2004, p. 11-2)

Esta regla estipula como ilegítima la relación comercial bajo condiciones distintas y más favorables que un país signatario del TLC pueda ofrecer a otro país que no forme parte del sistema de relación comercial establecido en el marco del Tratado.

Por su parte, en el artículo 11.4, se enumeran las disposiciones y regulaciones que se fijan para el acceso de las partes a los mercados, en cuatro aspectos que han sido incluidos en el inciso (a). Este inciso define el carácter de la liberalización comercial, que consiste en la eliminación total de restricciones de política económica o de cualquier otro tipo en cuanto a: a) "número de proveedores de servicios", b) "valor total de los activos o transacciones", c)"número total de operaciones de servicios o a la cuantía total de la producción de servicios", d) "número total de personas naturales que puedan emplearse en un determinado sector de servicios o que un proveedor de servicios pueda emplear y que sean necesarias para el suministro de un servicio específico y estén directamente relacionados con él". (SICE, 2004, p. 11-2)

Lo anterior, tal como queda precisamente establecido, señala que las relaciones contractuales comerciales entre los diferentes países involucrados en el Tratado, deberán respetar un juego de intercambio, el cual se sustenta privilegiadamente y con exclusión de cualquiera otra consideración, en el principio de la libre competencia y que, además, tiene como marco absoluto de referencia el trazo de la idealidad de un mercado donde, para decirlo en términos de una física analítico/mecanicista, la aceleración del intercambio no se vea interferida por fricciones de ningún tipo: legales, sociales, culturales, laborales, institucionales, etc. Asimismo, desde un punto de vista filosófico, podría decirse que la formulación que traza el inciso (a) del artículo 11.4, pasa igualmente a configurar algo así como la fundación de una ontología del mercado total. Este tipo de conceptualizaciones no es nuevo; antes bien, es ya recurrente y bastante prosaica. 
En su caso, el tema de la educación, en tanto tal, no aparece considerado en el TLC como parte de los servicios que, como en el caso de las telecomunicaciones, presentan un interés explícito particularmente delineado. Sin embargo, esto no quiere decir que la educación no esté implicada sustantivamente. $Y$ lo está en al menos dos sentidos principales: a) en la dimensión de la oferta y comercialización de servicios de educación superior en las modalidades a distancia, continua, virtual, etc.; b) en el ámbito del reconocimiento, acreditación y certificación de competencias y licencias para el ejercicio profesional. Al respecto, valga señalar que, en consecuencia, los cuatros aspectos considerados en el inciso (a) del artículo 11.4, le son concernientes a la educación superior de un extremo a otro.

\subsection{Oferta de servicios de educación superior}

En este primer sentido de su implicación en el TLC, la educación superior acusa una vinculación que la acerca y ubica en el contexto del componente de las telecomunicaciones. Desde Estados Unidos, durante los últimos años distintas instituciones de educación superior han venido desarrollando muy intensamente actividades en el campo de la oferta de servicios en línea (University of Phoenix, por ejemplo). (Cruz, 2004) Del mismo modo, también ha hecho aparición un nuevo tipo de establecimiento o entidad, las así denominadas universidades corporativas, tales como las que han sido creadas por las transnacionales Motorola, Coca Cola o Home Depot. (Cruz, 2004). Empresas estas que han optado por incursionar en el campo de la educación, desarrollando una oferta de servicios educativos especializada en lo que ahora se denomina como "educación para el trabajo"; actividades que involucran predominantemente ofertas de servicios de educación continua mediante la utilización de los recursos en red.

Adicionalmente, también es importante tener en cuenta la incursión y crecimiento acelerado de grupos transnacionales que están realizando inversiones significativas en el campo de la oferta de servicios de educación superior. Tal es el caso, por ejemplo, de los grupos Sylvan International Universities y Apollo Group Inc. El primero de estos grupos ha desarrollado una amplia acción de inversión en mercados extranjeros, que cubre servicios tales como: tutoreo en sedes, tutoreo por internet, enseñanza del inglés, capacitación de profesores, apoyo educativo, empresas de educación en línea, así como el establecimiento de campos universitarios en diferentes países del mundo. (Cruz, 2004).

La implicación de la oferta de servicios de educación superior en el TLC, sea bajo cualquiera modalidad que sea, representa condiciones inéditas para el desempeño y 
operación de las instituciones de educación superior locales, sobre todo en regiones como la de Centroamérica que, en su generalidad, presenta característicamente condiciones de gran debilidad en cuanto a inversión en investigación y desarrollo y, en su lugar, igualmente en materia de disposición y acceso a los recursos de las tecnologías de la información y la comunicación.

Así, según datos que aporta el Programa de las Naciones Unidas para el Desarrollo (PNUD) en su Informe sobre Desarrollo Humano para el año 2004, en el 2002 la inversión en Investigación y Desarrollo por parte de los Estados Unidos alcanzaba un 2,8\% del PNB; en su lugar, Costa Rica aparece registrando para ese año tan solo un $0,2 \%$ en este mismo rubro. PNUD, 2004, p. 180). Las relaciones para el conjunto de los países involucrados en el TLC se muestran en la tabla inserta a continuación.

Tabla 1: Estados Unidos y Centroamérica: inversión en ciencia y tecnología, en porcentajes del PNB, año $2002^{2}$

\begin{tabular}{|l|r|}
\hline \multicolumn{1}{|c|}{ País } & \% del PNB \\
\hline Estados Unidos & 2,8 \\
\hline Belice & $\cdots$ \\
\hline Costa Rica & 0,2 \\
\hline El Salvador & $\ldots$ \\
\hline Guatemala & $\cdots$ \\
\hline Honduras & $\ldots$ \\
\hline Nicaragua & 0,1 \\
\hline Panamá & 0,4 \\
\hline
\end{tabular}

Pero por otra parte, en un contexto de oferta de servicios educativos cuyas condiciones están determinadas como premisa por la disposición de recursos y plataformas tecnológicas altamente sofisticadas, resultan ostensiblemente débiles las condiciones de competitividad que serían accesibles para los proveedores locales en países como los de Centroamérica.

Considerando los costos de inversión, mantenimiento, actualización y producción de tecnología apropiada, las condiciones para la puesta en línea de los servicios educativos hacen emerger de nuevo las condiciones de debilidad existentes en cada uno de los diferentes países de la región. En su caso, la relación entre Estados Unidos y Costa Rica

\footnotetext{
${ }^{2}$ Fuente: Programa de las Naciones Unidas para el Desarrollo (2004), pp. 180-182.
} 
muestra de nuevo diferencias sustantivas. Con base en los datos del Informe sobre Desarrollo Humano 2004 ya citado, en el 2002 el número de ordenadores conectados a internet en Estados Unidos era de 551,4 por cada 1.000 habitantes -con un incremento de casi el doble con respecto al año $2000^{3}$-; en Costa Rica, esta misma relación es de 193,1. ${ }^{4}$

Tabla 2: Estados Unidos y Centroamérica: ordenadores conectados a internet, por cada 1.000 habitantes, año $2002^{5}$

\begin{tabular}{|l|r|}
\hline \multicolumn{1}{|c|}{ País } & $\begin{array}{r}\text { Ordenadores/cada } \\
\mathbf{1 0 0 0} \text { habitantes }\end{array}$ \\
\hline Estados Unidos & 551,4 \\
\hline Belice & 108,9 \\
\hline Costa Rica & 193,1 \\
\hline El Salvador & 46,5 \\
\hline Guatemala & 33,3 \\
\hline Honduras & 25,2 \\
\hline Nicaragua & 16,8 \\
\hline Panamá & 41,4 \\
\hline
\end{tabular}

Las diferencias en el desarrollo, producción y aplicación de las tecnologías de la información y la comunicación -cosa que no es para nadie un secreto-, reflejan en lo cuantitativo su significado en términos de repercusiones estructurales.

De este modo, en un escenario de libre interacción comercial como el que traza y busca organizar el TLC, la colocación en el mercado de los servicios educativos sustentados en el uso y aprovechamiento de las tecnologías de la información y la comunicación, en el mejor de los casos establecería ipso facto la configuración de una situación que, antes que estimular y potenciar las disposiciones y posibilidades para un desarrollo apropiado y autóctono, podría por el contrario provocar un efecto de inhibición sobre el desarrollo de este tipo de modalidades de prestación del servicio educativo en el marco de las instituciones de educación superior centroamericanas $\mathrm{y}$, en particular, por parte de las instituciones de educación superior pública. A propósito, cabe señalar que no resulta tan clara la validez que en su enunciación axiomática podría asistir a la idea de que, ante condiciones como las

${ }^{3}$ Cfr.: Programa de las Naciones Unidas para el Desarrollo (2002), 186-187.

${ }^{4}$ Cfr.: Programa de las Naciones Unidas para el Desarrollo (2004), p. 180

${ }^{5}$ Fuente: Programa de las Naciones Unidas para el Desarrollo (2004), pp. 180-182. 
descritas, los países pobres podrían beneficiarse "del aumento de las oportunidades y los avances del bienestar que genera el sistema multilateral de comercio" (Cuarta Conferencia Ministerial de la OMC, 2001), establecido en clave de libre mercado.

En razón de las regulaciones que propone el texto del Tratado, habría asimismo que tener en cuenta, en este sentido, la disposición que establece que ninguno de los países signatarios podría favorecer mediante subsidios ni mediante otro tipo de trato preferencial, las actividades que desarrolle un proveedor local de un servicio en cuya prestación estén interesados o involucrados proveedores de otros países. Tal como lo estipulan las reglas de "Trato Nacional" y de "Trato de Nación más Favorecida", las condiciones deben ser las mismas para todos los proveedores de todas las partes.

\subsection{Acreditación, certificación y reconocimiento de competencias profesionales}

El tema del reconocimiento de competencias profesionales, licencias y certificados, está asociado en el Tratado con las aptitudes, normas técnicas y prescripciones que se establezcan en relación con las actividades que realice un proveedor de otro país a nivel local en el territorio nacional.

El texto del Tratado especifica que la realización de este tipo de reconocimientos "podrá efectuarse mediante armonización o de otro modo, podrá basarse en un acuerdo o convenio con el país en cuestión o podrá ser otorgado de forma autónoma." (SICE, 2004, p. 11-4) Así, se identifican dos posibles vías para el otorgamiento del reconocimiento: a) mediante acuerdo o convenio con el país donde se obtuvo la educación o la experiencia profesional sometida a escrutinio, b) mediante procedimiento autónomo de reconocimiento. En cuanto a las medidas a adoptar:

...cada Parte procurará asegurar, como sea apropiado para cada sector específico, que cualquiera de tales medidas que adopte o mantenga:

(a) se basen en criterios objetivos y transparentes, como la competencia y la capacidad de suministrar el servicio;

(b) no sean más gravosas de lo necesario para asegurar la calidad del servicio; y

(c) en el caso de los procedimientos en materia de licencias, no constituyan de por sí una restricción al suministro del servicio. (SICE, 2004, p. 11-2)

En el caso de que un país disponga proceder mediante la aplicación de la vía que establece el reconocimiento de forma autónoma, de cualquier manera y según lo prescrito en el artículo 11.8, inciso segundo del Tratado, tal modalidad de reconocimiento deberá ser 
establecida de manera tal que las normas técnicas y prescripciones correspondientes, "no constituyan obstáculos innecesarios al comercio de servicios". (SICE, 2004, p. 11-2). Así:

Cuando una Parte otorgue el reconocimiento de forma autónoma, brindará a otra Parte las oportunidades adecuadas para que demuestre que la educación, experiencia, licencias o certificados obtenidos o los requisitos cumplidos en el territorio de esa otra Parte deban ser objeto de reconocimiento. (SICE, 2004, p. 11-2)

En este punto, según es evidente, hace su ingreso en la trama de las regulaciones del TLC, el tema de los estándares internacionales para la acreditación y la certificación, no sólo de competencias profesionales sino también de escuelas, carreras y programas. A propósito de la elaboración de normas y criterios para el otorgamiento de licencias y certificados a proveedores de servicios profesionales, tanto como sobre el reconocimiento mutuo de estas licencias y certificados, el texto del Tratado establece que las mismas podrán definirse y aplicarse a propósito de los siguientes aspectos:

(a) educación -acreditación de escuelas o de programas académicos; b) exámenes exámenes de calificación para la obtención de licencias, inclusive métodos alternativos de evaluación, tales como exámenes orales y entrevistas; (...) (e) desarrollo profesional y renovación de la certificación -educación continua y los requisitos correspondientes para conservar el certificado profesional. (SICE, 2004, p. 11-2).

Resulta aquí oportuno, no obstante, plantearse la cuestión acerca de quién establece un estándar de acreditación y desde dónde procede la donación de legitimidad que valida la utilización en común de ese estándar. Tal como, según lo anterior, resulta presumible, el tema del reconocimiento de licencias y competencias profesionales configura un vértice de intervención estratégica en la educación superior que, por la vía de la definición y homologación de indicadores y estándares, consecuentemente tendería a generar, o bien la creación de instancias ad hoc en el marco del Tratado, bien la creación de entidades transnacionales $\mathrm{o}$, en último término, tal como ha estado aconteciendo crecientemente durante los últimos años, a que sean las agencias de acreditación norteamericanas las que se hagan cargo de llevar a cabo los procesos y de otorgar los correspondientes títulos de acreditación y certificación.

Al respecto y, con todo, es necesario tener en cuenta que Estados Unidos es un país que posee una tradición institucional de más de cien años en materia de acreditación de escuelas, carreras y programas e instituciones de educación superior. En Centroamérica, en 
cambio, tal tipo de experiencias son por completo apenas emergentes y sólo ahora empiezan a ser desarrolladas de manera incipiente. Así, la propuesta del Consejo Superior Universitario Centroamericano (CSUCA) por crear un Sistema Centroamericano de Evaluación y Acreditación de la Educación Superior, tuvo su cristalización en 1998, recién hace poco más de un lustro. Asimismo, también es aún de más reciente creación el Consejo Centroamericano de Acreditación, que integra a diferentes actores (gobiernos, colegios y asociaciones profesionales, organismos de acreditación ya establecidos o en gestación, universidades públicas y privadas), como resultado de la Tercera Reunión del Foro Centroamericano de Acreditación, realizada en octubre de 2002.

En todo caso, se trata esta de una cuestión que alude a un escenario de relacionamiento completamente inédito, y que, además, demanda una urgente discusión por parte de los actores naturalmente interesados; en particular, por las instituciones de educación superior pública.

\section{TLC y Acuerdo General sobre Comercio de Servicios (AGCS)}

En la línea de las anteriores consideraciones, cabe señalar que puede ser considerado como un error significativo de apreciación, circunscribir el enfoque y el análisis del TLC en sus implicaciones para la educación superior, estrictamente al contexto específico que trazan las coordenadas de este Tratado.

Tal como se sabe y, sobre todo en las circunstancias que caracterizan los procesos estructurales de la globalización, la incidencia que tienen al menos algunos de los principales organismos multilaterales sobre los Estados/Nación y sobre la definición de las políticas públicas nacionales, puede llegar a tener expresiones a profundidad y de muy largo alcance. Ejemplo de ello lo son las experiencias precedentes de aplicación de los Programas de Ajuste Estructural (PAE's) que, para el caso de América Latina, condujeron a las realidades de la así eufemísticamente denominada "década perdida" del desarrollo de los años '90; así como también la incidencia que las políticas educativas nacionales de los últimos años en estos países (la polémica situación en Costa Rica, por ejemplo, en el caso de los exámenes nacionales de matemáticas), acusan como resultado de las "recomendaciones" realizadas en este campo por el Banco Mundial.

Los organismos multilaterales -de manera especial el Banco Mundial, el BID, o la Organización Mundial del Comercio (OMC)-, no necesariamente requieren disponer de acceso a mecanismos de incidencia directa ante los gobiernos o bien que les permitan interferir explícitamente en la definición de las políticas públicas nacionales. Al respecto, B. 
Bull (2001) señala que, en su lugar, tales organismos poseen a su disposición otro tipo de modalidades de intervención que les permite llevar a cabo con mucho éxito la canalización de sus políticas, objetivos y cursos de acción programáticos. Así, por ejemplo:

(i) A través de "policy based lending", donde las reformas forman parte de las condicionalidades para los préstamos;

(ii) A través de la cooperación técnica que en la mayoría de los casos incluye financiamiento para expertos extranjeros; $y$

(iii) A través del fortalecimiento a instituciones a largo plazo. (Bull, 2001)

En el marco de la Organización Mundial del Comercio (OMC), los resultados del proceso de negociaciones conocido como la Ronda de Uruguay, condujeron al establecimiento y entrada en vigor a partir de 1995 del Acuerdo General de Comercio de Servicios (AGCS). En este Acuerdo, existen contemplados doce sectores; uno de ellos, lo es la educación.

EI AGCS involucra, en su caso, aspectos y actividades concernientes a la educación superior, en la medida en que esta y los servicios que presta generan en la actualidad interés comercial en diversos sentidos:

...captación de estudiantes internacionales, establecimiento de campos universitarios en el extranjero, prestación de servicios con franquicia, aprendizaje en línea o electrónico, educación a distancia, universidades virtuales, sedes locales o campos satélites, instituciones gemelas, profesores e investigadores trabajando en el extranjero. (Knight, 2003)

\subsection{Un espacio de comercio promisorio}

Según información que aporta la Internacional de la Educación, la existencia de un escenario mundial de la educación superior que involucra gastos públicos que "sobrepasan ampliamente el billón de dólares" (IE, 2005), y que contempla "más de 50 millones de docentes, mil millones de alumnos y estudiantes, así como centenas de miles de establecimientos escolares diseminados por los cuatro puntos del globo" (IE, 2005), despliega la disponibilidad y existencia de lo que en el enfoque de algunos actores podría ser considerado como una especie de megamercado promisorio para el desarrollo privado y comercial de los servicios educativos en el marco de los TLC's; la apertura de las fronteras nacionales a la llamada "industria educacional" (Panizzi, 2002). 
A propósito, es pertinente traer a colación la afirmación realizada por el Banco Mundial en su documento de estrategia La educación en América Latina y el Caribe, donde considera como un logro impresionante de la educación superior latinoamericana, que un tercio de los estudiantes del tercer nivel estuviera al final de la década de los '90 matriculado en instituciones privadas. (Banco Mundial, 1999)

En el AGCS y, en lo que concierne a las actividades que involucran a la educación superior, destaca por sus características específicas el tipo de actividades definidas y clasificadas como tales. Estas incluyen los servicios educativos técnicos y vocacionales, así como "otros servicios de educación superior conducentes a título universitario o su equivalente." (Knight, 2003). Como nota importante a este respecto, J. Knight destaca que "los tipos de educación (i.e., negocios, estudios liberales, arte, ciencia) no están especificados. Se asume que están cubiertos todos los programas de capacitación y educación post-secundaria." (Knight, 2003).

Por su parte y según datos que ofrece C. García Guadilla, "a comienzos del 2002 existían 42 países -pertenecientes a la OMC-, que han realizado algún compromiso con al menos algún sector educativo. En ese grupo están incluidos todos los países de la OCDE menos Canadá, Finlandia, Islandia, Corea y Suecia." (García Guadilla, 2003).

En América Latina, de los tres países que han avanzado ya acuerdos para el comercio de servicios en el sector de la educación, releva el hecho de que dos de ellos sean países de Centroamérica: Costa Rica y Panamá. Por su parte, de los dos únicos países de América Latina que hasta ahora han suscrito acuerdos con respecto a comercio de servicios en el nivel de la educación superior, uno de ellos lo es también Panamá. (García Guadilla, 2002). De este modo, a nivel de América Latina y El Caribe, de los muy pocos países que hasta el momento han optado por inscribirse en el comercio de servicios educativos en el marco del AGCS, es oportuno destacar que dos de ellos se ubican paradójicamente en una de las regiones del mundo que, para la última década, presenta una situación caracterizada, o bien por el estancamiento o bien por el retroceso, en materia de inversión estatal en educación. (PNUD, 2004, pp. 172-174).

\subsection{Regulaciones homólogas}

Tomando en consideración las reglas del juego definidas en el marco del AGCS, aparece de nuevo la figura contractual que establece la regla de "Trato de Nación más Favorecida". Esta, según se ha indicado anteriormente, juega también un papel especial en las regulaciones estipuladas en el texto del TLC Centroamérica/Estados Unidos. 
Bajo el AGCS, si un país permite la competencia extranjera en un sector, se debe dar igualdad de oportunidades en dicho sector a los proveedores del servicio de todos los países miembros de la OMC. Esto también aplica al tratamiento de mutua exclusión. // Puede aplicar aún si el país no ha adquirido el compromiso específico de permitir el acceso de proveedores extranjeros a sus mercados. // Son permisibles las exenciones por un periodo de 10 años. // El NMF tiene implicaciones para aquellos países que ya están comprometidos con el comercio de servicios educativos y/o que permiten el acceso a proveedores extranjeros. (Knight, 2003).

Asimismo, también posee una relevancia especial la así denominada regla de la "Liberalización Progresiva":

El AGCS tiene una agenda incorporada, lo cual significa que con cada ronda de negociaciones hay mayor liberalización del comercio de servicios. Esto significa que se cubren más sectores y que se eliminan más restricciones al comercio. // Aplica a todos los sectores y por consiguiente incluye la educación. (Knight, 2003).

EI AGCS opera así con una agenda donde cada ronda de negociaciones involucra mayor liberalización, abarcando más sectores y eliminando mayores restricciones. "El proceso se lleva a cabo de "abajo hacia arriba", esto es, cada país determina el tipo y alcance de sus compromisos en cada sector, dentro de un contexto de liberalización progresiva." (García Guadilla, 2003).

Una característica importante que presentan estos procesos, la constituye el hecho de que las negociaciones dentro del AGCS las están asumiendo y llevando a cabo por regla general los ministros de comercio o de asuntos exteriores. (García Guadilla, 2003). En la mayoría de los casos, las negociaciones "se realizan con total desconocimiento de las partes interesadas en el sector que se negocia, como son -para el caso educativo- los Ministerios de Educación, las propias instituciones educativas, y los actores que forman parte de ellas (actores internos); y otros interesados en ellas (actores externos)." (García Guadilla, 2003).

La definición que incluye el AGCS con respecto a cuáles servicios se consideran como de carácter comercial, también es exactamente la misma que aparece en el artículo 11.1, inciso sexto, del TLC Centroamérica/Estados Unidos. De conformidad con lo estipulado en los acuerdos de 1994, dirigidos a la liberalización en escala planetaria del comercio "de todos los tipos de servicios" (Panizzi, 2003, p. 95), se estipula que no serían incluidos "entre los 
servicios reglamentados por la OMC aquellos suministrados en el ejercicio de la autoridad gubernamental y, en este contexto, no suministrados en base comercial." (Panizzi, 2003, p. 95).

No obstante, en 1998, en un documento declarado como confidencial, el secretariado de la OMC avanzó la tesis de que

como permiten la existencia de proveedores privados en la educación, los gobiernos aceptan el principio de que la educación, en especial la educación superior, puede ser tratada como un servicio comercial y, por lo tanto, deba ser reglamentada en el cuadro de la OMC. (Panizzi, 2003, p. 95).

Asimismo, en octubre de 1999, de nuevo el secretariado de la OMC definió formalmente los servicios reglamentados por el AGCS, dentro de los cuales dispuso incluir también el de la educación.

A partir del 2000, la OMC inició negociaciones visando la liberalización de los servicios educativos. Durante este mismo año, Estados Unidos, Australia y Nueva Zelanda "proponen a los otros países una apertura prácticamente sin límites de sus mercados" a los proveedores oriundos de sus territorios y solicitan que toda la restricción a la acción de esos grupos por los gobiernos nacionales sea rechazada". Como nos muestra Marco Antonio Rodríguez Días, a pesar de que un país como Japón había presentado serias restricciones a esas medidas, la movilización en favor de su adopción por el conjunto de los Estados Miembros de la OMC es grande. En definitiva, el proceso de transformación de la educación en servicio comercial está en curso... (Jouen y otros, 2004).

Así, muy recientemente, en la reunión del Consejo General de la OMC realizada en julio de 2004, se establecieron nuevos plazos para el proceso del AGCS.

Se acordó que todos los países que cuenten con solicitudes de parte de otros Estados miembros a fin de entablar más compromisos en servicios específicos deben examinar y mejorar sus ofertas antes del 1 de mayo de 2005. (Jouen y otros, 2004).

A criterio de la Internacional de la Educación:

Este plazo representa una mayor presión, especialmente para los países en desarrollo que todavía no han presentado ofertas, para que lo hagan y a fin de que todos los países en general mejoren la "calidad" de sus ofertas. (Jouen y otros, 2004). 
En tal sentido y, no obstante, cabe interrogarse hasta qué punto lo anterior resulta compatible con lo que los propios gobiernos también han acordado y suscrito en otro tipo de espacios igualmente de carácter multilateral. Al respecto, vale destacar uno de los enunciados incluido en la Declaración de Bariloche, suscrita por los jefes de Estado y de gobierno de los países participantes en la $V$ Cumbre de la Organización de Estados Iberoamericanos para la Educación, la Ciencia y la Cultura:

El acceso del conjunto de la población a los valores, conocimientos y competencias que brinda el sistema educativo se constituye en un elemento imprescindible para garantizar la continuidad y permanencia de las instituciones democráticas, la participación política, económica, social y cultural, en particular para los grupos más desposeídos, y como parte de la lucha contra la pobreza. (V Cumbre Iberoamericana de Jefes de Estado y de Gobierno, 1995).

Como uno de los principios rectores estipulado con carácter de relevancia especial para la verificación del compromiso que los gobiernos acceden a asumir con la educación, en esa misma Declaración se señala adicionalmente que: "Las políticas educativas deben ser políticas de Estado, basadas en el consenso y la participación de todos los sectores sociales, a fin de garantizar el acceso a la educación a toda la población sin distinción alguna." (V Cumbre Iberoamericana de Jefes de Estado y de Gobierno, 1995).

Aún cuando, a propósito de las directrices que se proponen establecer como contenidos de negociación, en su caso, en el marco del TLC Centroamérica/Estados Unidos, los enunciados anteriores evidentemente podrían permitir la apertura de un rango relativamente amplio de flexibilidad semántica para apoyarse en ella y oponerla como un "contrafáctico conceptual" ante emergentes y eventuales objeciones, también es cierto que, en su lugar, esos mismos enunciados no dejan de establecer una ruta de significación a través de la cual se precisa la importancia fundamental que los gobiernos otorgan a la educación, como soporte que sustenta las posibilidades de la democracia tanto como las condiciones del desarrollo social y sostenible.

\subsection{Implicaciones para la educación superior}

En la dimensión de sus configuraciones más concretas, si quien pasa a determinar ahora y cada vez con mayor intensidad cuáles son las necesidades de formación requeridas son los actores y fuerzas del mercado a través de procesos y escenarios como los del TLC, tales entidades podrían igualmente disponer a nivel nacional el establecimiento de ofertas 
educativas transnacionalizadas, o bien la presencia de sedes de universidades extranjeras que ofrezcan el tipo de servicios y modalidades de formación deseados, sin mayor consideración de lo que todo esto pudiera representar en términos de la calidad y excelencia de la educación, por no hablar de las repercusiones estructurales adversas que, a la larga, tales disposiciones y coordenadas también podrían llegar a tener en su conjunto para los sistemas y las instituciones de educación superior.

Por su parte, inscritos en escenarios de regulación comercial multilaterales y de escala planetaria como el del AGCS, los gobiernos y Estados han acordado suscribir los acuerdos de un proceso que conduce a adquirir compromisos en materia de comercio de servicios educativos, al margen de las consideraciones que al respecto puedan ser planteadas por las propias instituciones nacionales que tienen a su cargo específicamente la competencia sobre estos asuntos.

La legislación y las respectivas disposiciones constitucionales existentes a escala nacional, según esto, no tendrían necesariamente por qué ser interpeladas ni involucradas; una estrategia de convenios específicos y ubicados en espacios puntuales podría ser suficiente.

No obstante y, en todo caso, los servicios de educación superior pública, afrontarían con ello una situación compleja que podría interferir con la configuración de las condiciones para el desarrollo de sus respectivos proyectos académicos e institucionales.

Las tecnologías de la información y la comunicación, operadas en su máximo posible de aplicación a propósito de una oferta de servicios de educación superior en clave de comercialización transnacionalizada, posibilitan la apertura de los espacios institucionales a la desterritorialización y reterritorialización ${ }^{6}$ de las prácticas académicas, los estilos y culturas de la gestión, tanto como asimismo de las modalidades y estrategias para la prestación del servicio educativo y para la organización y desarrollo del proceso de formación.

Asimismo y, muy probablemente, en un contexto histórico/social donde la realidad nacional de la demanda de servicios educativos está atravesada por la masificación y por la exigencia de contar con un título para obtener una posibilidad de inserción en el mercado de trabajo, favorece que propuestas como las del AGCS puedan llegar a tener en lo inmediato una acogida favorable. En un marco de expansión del sector de los servicios y de eclosión de la demanda de servicios de formación en el nivel de la educación superior, los

\footnotetext{
${ }^{6}$ Utilizando estas expresiones en el sentido aproximadamente en que lo hacen Deleuze/Guattari (2000) en Rizoma, p. 23. Véase también: Guattari, Félix (1995), p. 208.
} 
argumentos para defender la preeminencia y operación exclusiva de los servicios educativos nacionales podrían resultar vulnerables y en la práctica de escasa recepción por parte de los actores interesados.

\section{Reacciones en América Latina}

Es oportuno recalcar el hecho de que, en el marco del AGCS y, como disposición procedimental común, las tendencias impulsadas y los procesos puestos en marcha, no necesariamente están pasando por el escrutinio de los sistemas y las instituciones de educación superior; quienes están llevando a cabo las negociaciones son directamente los gobiernos, a través de sus Ministerios de Economía o de Relaciones Exteriores. Por regla general, los representantes del sector educativo no tienen participación en las negociaciones.

En la "Carta de Porto Alegre", resultado de la III Cumbre Iberoamericana de Rectores de Universidades Públicas realizada en abril de 2002, se señala que el AGCS:

...lesiona seriamente las políticas de equidad indispensables para el equilibrio social, en especial para los países en desarrollo, necesarias para corregir las desigualdades sociales, y tienen serias consecuencias para nuestra identidad cultural. Perturba igualmente la consolidación y transmisión de valores éticos y culturales y afecta nuestras aspiraciones de lograr una sociedad más democrática y justa a través de un desarrollo sostenible. Aspectos todos ellos a los que contribuye la educación superior, cuya misión específica se define en virtud de una concepción de bien social público, destinada al mejoramiento de la calidad de vida de nuestros pueblos, función que en ningún caso puede cumplir si se la transforma en simple mercancía, u objeto de especulación en el mercado, a través de su comercialización internacional. Por último, entre los graves problemas que esta circunstancia acarrea, tenemos que mencionar la uniformización acrítica de la educación y el grave daño que significa para la soberanía nacional y de los pueblos. (García Guadilla, 2003).

Los márgenes de maniobra de que disponen las instituciones de educación superior pública, no obstante, ante las incidencias que en las políticas públicas nacionales generan el TLC y el AGCS, podrían verse minimizados a lo que, por su parte, en una observación autocrítica que interpela y convoca a una toma de posición y al desarrollo de las acciones correspondientes, G. Neave identifica como simples manifestaciones de intenciones (Neave, 2001, p. 38). Es decir, el escenario planteado y su proceso de configuración emergente, 
interpela a ir más allá de una disposición reactiva, o de acciones que al no estar concretamente fundadas ni organizadas institucionalmente, no alcanzan a representar mayores efectos y alternativas que pudieran tener una incidencia significativa sobre la configuración de las situaciones que derivan como resultado del despliegue de los nuevos escenarios y condiciones estructurales devenidos.

Más allá de lo que pueda acontecer a futuro en el escenario de desarrollo del AGCS y, en su caso, de lo que los procesos del TLC puedan llegar a representar para la educación superior pública en países como los de la región centroamericana, lo cierto es que, al menos de momento, el proceso que tiende a la comercialización se nutre y fortalece de las propias condiciones internas de debilidad. En un caso como el de Centroamérica, tales condiciones de debilidad están dadas por los siguientes factores y tendencias, que esta región comparte igualmente con otras regiones del mundo en condición de desventaja:

-El uso creciente de TIC's para la enseñanza de programas a nivel nacional y más allá de las fronteras.

-El creciente número de entidades privadas con ánimo de lucro que ofrecen educación superior a nivel nacional e internacional.

- Las matrículas y otros costos cada vez más altos a los que se enfrentan los estudiantes de instituciones públicas (y privadas).

- La necesidad que tienen los proveedores públicos de buscar fuentes alternas de financiación, lo que a veces significa participar en actividades lucrativas o buscar el apoyo financiero del sector privado.

- La habilidad del gobierno para financiar la creciente demanda de educación superior y para adultos. (Knight, 2003).

Las orientaciones y avances que el escenario del AGCS involucra, obligan en consecuencia a las instituciones de educación superior pública a prestar mayor atención al proceso y a definir una posición clara al respecto. Como es evidente, también afrontan la exigencia de establecer los espacios y dispositivos necesarios de investigación y de acopio de información, que permitan identificar, en específico y estructuralmente, de qué manera se vería la sociedad en su conjunto afectada por estas nuevas tendencias.

Está claro que las instituciones de educación superior pública, no son instituciones que puedan tomar decisiones con independencia de los intereses de las instituciones y las fuerzas más dinámicas de la sociedad. En este sentido, no las acuerpa y, en su lugar, más bien están distantes de la naturaleza de las entidades empresariales, las cuales, en principio, 
pueden organizarse y dar a sus proyectos la orientación que el criterio de la tasa de ganancia les indique como la más adecuada. Antes bien y, por el contrario, las instituciones de educación superior pública, cumplen con un papel muy especialmente significativo: ellas representan los intereses de la sociedad en lo que a formación de excelencia, producción de conocimiento y mejoramiento de la calidad de vida se refiere (González García, 2004, p. 3); y tienen la responsabilidad de representar y asumir, en este sentido, las expectativas, intereses y necesidades de los diversos sectores que integran el entramado social.

Tal como lo señala J. Knight, ante el escenario en el que la educación superior pública queda inscrita como parte del proceso del AGCS, se establece la necesidad "de que el sector educativo analice estas inquietudes y las consulte con las partes interesadas. Al mismo tiempo, es necesario ser proactivo y estratégico al supervisar e influenciar la posición del gobierno en las negociaciones en la etapa de solicitud/oferta del AGCS." (Knight, 2003).

Frente a ciertas demandas e intereses de la sociedad, que en la actualidad manifiestan una ostensible orientación a valorar una formación centrada en las habilidades operacionales para el mundo del trabajo y la economía, la educación superior debe concretamente desarrollar propuestas de formación que no necesariamente queden restringidas a la orientación que tales demandas involucran.

\section{Conclusiones}

Interesa recalcar en la consideración de que, tal como lo muestran los elementos de análisis aquí considerados, existe una íntima vinculación entre los lineamientos y procesos del AGCS, por una parte, y lo que, por la otra, constituyen los elementos de articulación del escenario de intercambio comercial trazados en el documento de propuesta de TLC entre Centroamérica y Estados Unidos. En sus orientaciones e implicaciones estructurales, puede afirmarse que se trata exactamente del mismo proceso; únicamente que articulado en dos escalas diferentes. La primera de ellas, la constituye como tal la escala ampliada y multilateral del AGCS; la segunda, la del TLC, remite a un escenario de negociación localizado geográficamente de manera específica.

A propósito de lo anterior, vale dar aquí una referencia especial a lo que los gobiernos de los países miembros de la OMC señalan y suscriben en la Declaración Ministerial de Doha:

Recalcamos nuestra fidelidad a la OMC como único foro para la elaboración de normas comerciales y la liberalización del comercio a escala mundial, reconociendo asimismo que los acuerdos comerciales regionales pueden desempeñar un papel 
importante en la promoción de la liberalización y expansión del comercio y en el fomento del desarrollo. (OMC, 2001).

Las instituciones de educación superior pública, tal como lo recalca M. Panizzi, son conscientes de que:

...el debate académico y la investigación científica no conocen fronteras. Sin embargo, debido a la iniciativa de la OMC, están frente a un desafío de otra naturaleza. No está en cuestión, en este caso, la formación de redes de investigación, la movilidad docente y estudiantil u otras formas de intercambio científico y cultural siempre valorizadas por nosotros. Lo que está en cuestión, y lo que interesa a casi todos en casi todos los países del mundo, es el destino mismo del conocimiento como patrimonio social y de la educación como bien público. (Panizzi, 2003, p. 96).

Ante la puesta en cuestión, especialmente de la educación y de la educación superior como bien público, es importante relevar la condición de que estas instituciones cuentan a su disposición con una diversidad de mecanismos y espacios de autonomía que les permiten llevar a cabo un desarrollo informado y crítico de su interactuación con la sociedad. De este modo, disponen de los espacios y condiciones necesarios para interpretar y tomar una posición informada, apropiada y prospectiva, frente a los procesos y configuraciones de sentido político y económico que, como en el caso del AGCS y del TLC, repercuten en las condiciones del desarrollo nacional estratégico. Es decir, las instituciones de educación superior cuentan con condiciones institucionales que les permiten interpretar tales procesos y tendencias con base en sus propios criterios informados y, en este sentido, replantearse crítica, reflexiva y propositivamente el sentido de las demandas que, o bien la sociedad en su conjunto o bien los agentes, actores y organismos que operan a través de esta y de sus instituciones, les plantean y establecen como referente de orientación programática. (Barnett, 2001, p. 40).

En su caso, los estancamientos o atenuación de ritmos que eventualmente se hayan podido presentar en el transcurso del proceso de liberalización comercial impulsado a partir de 1995, en la escala ampliada y multilateral del AGCS y, particularmente como resultado de la intervención de los actores que se oponen a este proceso, en la escala geográfica localizada del TLC podrían antes bien ser resueltos mediante procedimientos más expeditos y a través de "negociaciones" que reducen la complejidad del escenario en lo que a la participación de diversos actores se refiere. 
Las instituciones de educación superior pública de Costa Rica y, desde los distintos lugares en que se configuran y articulan sus comunidades académicas, durante los últimos meses han dispuesto llevar a cabo diversas actividades dentro de un proceso de análisis en torno al TLC. En esas actividades ha tenido lugar la participación de múltiples actores institucionales internos y externos. No obstante, este proceso requiere ser profundizado y ampliado aún más; sobre todo, es necesario intensificar y completar la fase de análisis y diagnóstico en la que se ha estado hasta ahora, para transitar a la fase de elaboración de propuestas y de identificación de cursos de acción que establezcan el desarrollo de una mayor capacidad institucionalizada de alcance e incidencia.

En procura de abonar a tales acciones y procesos, la pertinencia de estudios sobre la educación superior y de sus relaciones con los procesos, tendencias y esquemas operacionales de negociación del libre comercio, configuran una necesidad a la que es preciso continuar dando una atención ampliada y diversificada.

\section{Referencias}

Alarcón Alba, F. y J. Luna. (2003). Acreditación y evaluación de la educación superior en Centroamérica. Recuperado el 15 de junio de 2004, http://iesalc.unesco.org.ve/acreditacion/centroamerica/acr cam alarcon.pdf

Banco Mundial. (1999). Documento estratégico del Banco Mundial: la educación en América Latina y el Caribe. Recuperado el 11 de julio de 2004, http://wbln0018.worldbank.org/LAC/lacinfoclient.nsf/d29684951174975c85256735007f ef12/e0edf9d64c461e5185256968006bfddc/\$FILE/Spanish-entire.pdf

Barnett, Ronald. (2001). Los límites de la competencia. El conocimiento, la educación superior y la sociedad. Barcelona: Gedisa.

Bull, Benedicto. (2001). Ponencia para Encuentro Internacional Ética y Desarrollo: los nuevos desafíos. Recuperado el 25 de junio de 2003, http://www.iadb.org/etica/documentos/ho bul etica.doc

Conferencia Mundial sobre la Educación Superior. (1998). La educación superior en el siglo XXI. Visión y acción. París: Ediciones UNESCO.

Comisión Económica para América Latina y El Caribe. (2000). Equidad, desarrollo y ciudadanía. Versión definitiva. Recuperado el 7 de enero de 2003, http://www.cepal.cl/publicaciones/SecretariaEjecutiva/1/lcg2071/lcg2071.pdf.

Cruz, Carlos. (2004). Inversión extranjera en educación privada con fines de lucro. Recuperado el 30 de octubre de 2004, http://www.anuies.mx/index1024.html 
Cuarta Conferencia Ministerial de la OMC. (2001). Declaración Ministerial. Recuperado el 1 de marzo de 2005, http://www.wto.org/spanish/thewto s/minist s/min01 s/mindecl s.htm

Deleuze, Pilles; Félix Guattari. (2000). Rizoma. Introducción. $3^{a}$ ed. Valencia: Pretextos.

García Guadilla, Carmen. (2003). Acuerdo General de Comercialización de Servicios (GATS) y educación superior en América Latina. Algunas ideas para contribuir a la discusión. Recuperado el 2 de febrero de 2004, http://www.columbusweb.com/es/partej/gatsyal.doc

García Guadilla, Carmen. (2003). Balance de la década de los '90 y reflexiones sobre las nuevas fuerzas de cambio en la educación superior. En: Mollis, Marcela (comp.) Las universidades en América Latina: ¿reformadas o alteradas? La cosmética del poder financiero. Buenos Aires: CLACSO.

González García, Yamileth. (2004). La universidad que somos y que queremos ser. San José, en prensa.

Guattari, Félix. (1995). Cartografías del deseo. Buenos Aires: La Marca.

Internacional de la Educación/Internacional de los Servicios Públicos. (2005). La OMC y la Ronda del Milenio: los retos de la educación pública. Cuestiones de interés común para educadores y trabajadores del sector público. Recuperado el 4 de marzo de 2005, http://www.ei-ie.org/pub/spanish/spbei-psi wto.html

Jouen, Elie y otros. (2004). Educación versus comercio. Recuperado el 4 de marzo de 2005, http://www.ei-ie.org/pub/spanish/tradeducation/tradeducation 03 s.pdf

Knight, Jane. (2003). Comercialización de servicios de educación superior: implicaciones del AGCS. Recuperado el 2 de febrero de 2004, http://www.columbus-web.com/es/partej/reportgats.doc

Neave, Guy. (2001). Educación superior: historia y política. Estudios comparativos sobre la universidad contemporánea. Barcelona: Gedisa.

Panizzi, Wrana María. (2003). La educación superior como "servicio comercial": ¿Desafío o amenaza? En: García Guadilla, Carmen (ed.) El difícil equilibrio: la educación superior como bien público y comercio de servicios. Implicaciones del AGCS (GATS). París: Programa Columbus.

Programa de las Naciones Unidas para el Desarrollo. (2002). Informe sobre desarrollo humano 2002. Profundizar la democracia en un mundo fragmentado. Recuperado el 15 de octubre de 2003, http://www.desarrollohumano.org.sv/informeMundial2002/indicadores\%208.pdf.

Programa de las Naciones Unidas para el Desarrollo. (2004). Informe sobre desarrollo humano 2004. La libertad cultural en el mundo diverso de hoy. Recuperado el 20 de febrero de 2005, http://hdr.undp.org/reports/global/2004/espanol/pdf/hdr04 sp complete.pdf\#search='i nforme\%20desarrollo\%20humano\%202004 
Rosenthal, Pert. (1998). Los desafíos de la globalización para Centroamérica. $\begin{array}{llllll}\text { Recuperado el de } 4 \text { abril } & \text { de }\end{array}$ http://www.cepal.cl/publicaciones/SecretariaEjecutiva/7/lcg2037/rosenth.htm.

Sistema de Información sobre Comercio Exterior. (2004). Texto del Tratado de Libre Comercio entre República Dominicana, Centroamérica y los Estados Unidos. Recuperado el 4 de febrero de 2005, http://www.sice.oas.org/Trade/CAFTA/CAFTADR/CAFTADRin s.asp.

V Cumbre Iberoamericana de Jefes de Estado y de Gobierno. (1995). Declaración de Bariloche. Recuperado el 30 de noviembre de 2003, http://www.campusoei.org/oeivirt/fp/cuad1a02.htm 